\title{
Are Mispricings Long-Lasting or Short-Lived? Evidence from S \& P 500 Index ETF Options
}

\author{
Feng Jiao \\ University of Lethbridge, Calgary, Canada \\ Email: feng.jiao@uleth.ca
}

How to cite this paper: Jiao, F. (2018) Are Mispricings Long-Lasting or Short-Lived? Evidence from S \& P 500 Index ETF Options. Theoretical Economics Letters, 8, 378-389. https://doi.org/10.4236/tel.2018.83027

Received: December 26, 2017

Accepted: February 9, 2018

Published: February 12, 2018

Copyright $\odot 2018$ by author and Scientific Research Publishing Inc. This work is licensed under the Creative Commons Attribution International License (CC BY 4.0).

http://creativecommons.org/licenses/by/4.0/

\begin{abstract}
A rapidly growing literature has documented evidences suggesting the mispricing of options. Building on recent results of option pricing bounds imposed by stochastic dominance, this paper examines the time-series proprieties of such mispricing. In an application to high-frequency bid/ask quotes of S \& P 500 index ETF options, this paper provides evidences that most violations of the stochastic dominance bounds last no more than 10 trading hours. The typical duration of mispricing is even shorter for near to maturity options. The results imply that the observed widespread mispricing in options might be the result of temporary inefficiency (e.g. transaction costs, overreaction, liquidity etc.) rather than a model misspecification, such as estimation biases of the parameters, or an overlooked persistent risk factor.
\end{abstract}

\section{Keywords}

Stochastic Dominance, Index Option, High-Frequency Trading

\section{Introduction}

Finance literature documents a number of evidences suggesting the mispricing of options. Given a steep smile in the implied volatility of S \& P 500 index option, the out-of-the-money (OTM) options seem to be expensive [1] [2]. For example, shorting the zero beta straddles/strangles offered a return of 3.15 percent per week [3] [4]. Also, widespread violations of stochastic dominance by 1-month S \& P 500 index call options imply that any risk-averse trader can improve expected utility by writing call options net of transaction costs and bid-ask spread [5] [6] [7]. Santa-Clara and Saretto [8] find that strategies involving short positions in options generally compensate the investor with Sharpe ratios as high as 1.69 . 
In spite of a large body of literature attempting to identify the mispricing of options, questions still remain: are mispriced options always mispriced until maturity? If not, how long does the mispricing period last? How often do options move from mispriced to fairly priced and vice versa?

Answering these questions may shed light on the underlying mechanism of such mispricing. If the "mispricings" were the results of model mis-specifications, e.g. unknown risk factors, or errors in estimating parameters, they should be present for a long duration, as the flawed pricing models generate systematic pricing biases. If such "mispricings" were simply market temporary inefficiency due to market frictions or overreactions, they should be short-lived as arbitragers can take advantages of such opportunities quickly. The answer of long-lasting or short-lived mispricing is also crucial to practitioners since it determines how soon the arbitrage strategies will pay off. If prices converge towards fair value slowly, it may take too long to realize any profit.

This paper investigates the time series properties of option mispricings using high frequency bid-ask prices. After constructing option pricing bounds based on stochastic dominance, this paper provides evidences that most violations of the stochastic dominance upper bounds of Constantinides and Perrakis [7] last no more than 10 trading hours. This study also identifies that options move in and out of the pricing bounds frequently during the last few days before maturity. The results are robust to the different parameters and assumptions in estimating the bounds.

This paper contributes to the literature by showing that mispricings in options are mostly short-lived. This means that the observed widespread overpricing in options might be the result of temporary inefficiency (e.g. transaction costs, overreaction, liquidity etc.) rather than a model mis-specification, such as estimation biases of the parameters, or an overlooked persistent risk factor. It supports the option pricing bounds derived in Constantinides and Perrakis [7].

The dataset used in this study differs from prior literatures. Most existing studies are based on the historical end-of-day mid prices of index options, retrieved from Option Metric database. The data used in this study are unique in two ways. Firstly, this study chooses S \& P 500 index ETF (SPY: NYSE) as the underlying security, as they have high liquidity and small bid-ask spread. Secondly, the dataset in this study comes from Interactive Broker trading platform. With a real-time electronic trading platform, it provides live bid/ask quotes synchronized with AMEX, CBOE and other large exchanges. The observations in this dataset come from the historical snapshots of the quotes every fifteen minutes. Compared to traditional Option Metrics database, the datasets contain bid/ask quotes with high frequency and are of higher quality. Due to limitation of the database, the sample period is restricted to half a year. However, the total observations amount to over 40,000 quotes.

The paper is organized as follows. The first section presents the pricing bounds on option prices imposed by stochastic dominance as in Constantinides and 
Perrakis [7] and examines the underlying assumptions. The next section describes the data and the experiment design. The empirical results are shown in Section III and Section IV checks their robustness. In the last section of this study, I discuss the implications of the results and conclude.

\section{Option Pricing Bounds Imposed by Stochastic Dominance}

Constantinides and Perrakis [7] investigate the restrictions on option prices imposed by stochastic dominance. They conclude that options prices should stay within a set of bound in equilibrium. Otherwise, any trader can increase expected utility by trading in the options, the index, and the risk-free bond - hence violates the conditions of equilibrium.

In this study, the term "mispricing" is defined as the option prices which violate the restrictions in Constantinides and Perrakis [7].

\subsection{Assumptions to Derive the Option Pricing Bounds}

To derive the option pricing bounds, Constantinides and Perrakis [7] assumes that the utility-maximizing and risk-averse agents are capable to hold and trade only two representative securities in the market, a stock index and a bond. Stock trades incur proportional transaction costs.

They search for the possible prices of the bond, stock, and derivatives at a given point such that those prices support an increasing and concave utility function. If the combination of prices fails to support the utility function, then any trader can increase expected utility by trading in the options, the index, and the riskless asset. The violation of such bounds are called inconsistent with stochastic dominance as it implies that at least one risk-averse agent, regardless of the form of utility function, increases expected utility by trading the options.

\subsection{Pricing Bounds on Call Options}

This section presents the pricing bounds on call options without proof. At any time $t$ prior to expiration $T$, the upper bound on the price of a call option is given by

$$
\bar{c}\left(S_{t}, t\right)=\frac{1+k}{(1-k) R_{S}^{T-t}} E\left[\max \left(S_{T}-K, 0\right) \mid S_{t}\right]
$$

where $S_{t}$ is the underlying price at time $t, K$ is the strike, $k$ is the proportional transaction costs, and $R_{S}$ is the expected return of the underlying per period.

For the lower bound,

$$
c\left(S_{t}, t\right)=(1+\delta)^{t-T} S_{t}-\frac{K}{R_{f}^{T-t}}+\frac{E\left[\max \left(K-S_{T}, 0\right) \mid S_{t}\right]}{R_{S}^{T-t}}
$$

where $R_{f}$ is the gross risk-free return per period, and $\delta$ is the dividend yield.

Constantinides and Perrakis [9] also derived the option pricing bounds imposed by stochastic dominance on put options. However, empirical evidences suggest that the violations of bounds on puts are sparse [6]. Consequently, this 
research limits our focus on the violations of option pricing bounds on call option.

\section{Data and Methodology}

This research intends to find the mispricings implied by upper/lower bounds of option prices, and to examine the time series properties of such violations. This section first presents the methodology and dataset to construct the option pricing bounds. Then, we discuss about the criteria to identify the mispricings when both ask and bid price are present.

\subsection{Estimation of Option Pricing Bounds}

There are three steps involved to implement the empirical test: estimating input parameters and distribution; feed into Equation (1) and (2) to derive the upper/lower bounds; compare them with market prices determine the time series pattern of violations.

Input Parameters and Return Distribution. To calculate the option pricing bounds as stated in the previous section, the primary challenge is to estimate the conditional distribution of the underlying index return. This paper employs several techniques to achieve the task: bootstrapping, GARCH model, and adjusted Chicago Board Options Exchange Volatility (VIX) index.

The first approach is to bootstrap from one month (22 trading days) overlapping index returns with a rolling window of six months (132 trading days), such that each day is the beginning of another one-month return. This is a widely accepted approach in financial investment industry.

In the second way, the conditional distribution comes from the forecast of $\operatorname{ARMA}(1,1)-\operatorname{GARCH}(1,1)$ model with error terms distributed as skewed student's $t$. An $\operatorname{ARMA}(m, n)-\operatorname{GARCH}(p, q)$ process model the index return as a stationary $\operatorname{ARMA}(m, n)$ process, and the conditional volatility as a $\operatorname{GARCH}(p$, $q)$ process. The deviation from traditional assumptions of normal distributed error terms allows for the negative skewness and excessive kurtosis observed in actual index return.

The third estimation approach parallels the bootstraps approach, but rescales the distribution such that the volatility of expected returns matches the adjusted VIX index. This method adopts the VIX index as the benchmark volatility because VIX reflects the market expectation of one-month ahead volatility. The rationales of the adjustment of VIX index are that VIX generally overpredicts the realized volatility. As a result, the last approach sets the adjusted VIX as the fitted values of regressing VIX index on realized volatility ${ }^{1}$.

The input parameters used to calculating the option pricing bounds are summaries as follows (Table 1$)$. Risk Free Rate $\left(R_{f}\right)$ is chosen as the three-month

${ }^{1}$ The VIX is usually higher than realized volatility. To adjust VIX, I run a linear regression of VIX on realized volatility. This relationship is stable across time. As a result, throughout all the time periods, I use VIX $_{\text {adj }}=0.958 *$ VIX -2.472 as one of the input to model the return volatility. 
T-bill Rate, with an average of $0.18 \%$ during the sample period. Dividend Yield $(\delta)$ parameter is retrieved from market S \& P 500 Dividend Yield, with a mean of $2.14 \%$ during the sample period. The proportional transaction Cost $(k)$ is fixed at $0.3 \%$. This is based on the best estimation of two senior derivatives traders with more than 10 years of experiences in an assets management firm.

Calculation of Pricing Bounds. Finally, after estimating the statistical distribution of index return, the calculation of the term $E\left[\max \left(S_{T}-K, 0\right) \mid S_{t}\right]$ in equation (1) and (2) requires Monte Carlo simulation techniques. Control variants and antithetic variants techniques are employed in Monte Carlo simulations to reduce the variance. The typical number of replications is $800,000^{2}$.

Criteria of Determining Mispricings. As this research studies the typical duration of option mispricings, it necessitates the criteria to characterize mispricings and reasonably priced options. The definition of mispricings is straightforward. When both bid and ask prices are present, the under-pricing is defined as Ask Price < Lower Bound, and overpricing is identified if Bid Price > Upper bound. Because the ticker size in CBOE is set at $\$ 0.01$, this requires that ask price should be less than the lower bound by over $\$ 0.01$ to be qualified as under-pricing. Similar condition applies to overpricing.

The criteria determining the reasonably priced options are a little tricky when both bid and ask prices present. When the ask price goes below the upper bound and the bid goes beyond the lower bound, it is unambiguous to claim such

Table 1. An example of the input parameters.

\begin{tabular}{ccc}
\hline Parameters & Methods & Estimation \\
\hline Volatility & Bootstrap (Past 126 Days) & $13.36 \%$ \\
ARMA(1,1)-GARCH(1,1) & $17.27 \%$ \\
Skewness & Adjusted VIX & $17.86 \%$ \\
& Bootstrap (Past 126 Days) & -0.094 \\
ARMA(1,1)-GARCH(1,1) & -0.046 \\
Risk free rate $\left(R_{F}\right)$ & Adjusted VIX & -0.094 \\
Dividend yield $(\delta)$ & 3-months T-bill Rate & $0.09 \%$ \\
Transaction cost $(k)$ & Market S \& P 500 Dividend Yield & $2.20 \%$ \\
\hline
\end{tabular}

*Source: Author's computation. This table describes an example of the input parameters to calculate the bounds on June-08-2012 at 4:00 PM. The first part of table summarizes the statistics of estimated return distribution under three different approaches. The last part of the table provides other input parameters. The adjusted VIX approach is the same as the Bootstrap, except rescaling the distribution to match the variance to the adjusted VIX. T-bill Rate is obtained from U.S. Department of Treasure, other data are from Interactive Broker trading platform.

${ }^{2}$ One concern of the Monte Carlo simulation is that it may lead to frequent short term violations of the bounds, since the bounds may fluctuate across time simply because of the sampling errors. To address this issue, the simulation in this paper employs a fixed seed in the random number generator. Thus, this procedure could only bias the duration of the violations upward, as the sampling errors persist through time. 
option is fairly priced. However, if ask price exceeds the upper bounds while bid sits between the upper and lower bounds, it is unclear whether such option is mispriced. Although the ask price appears to be overpriced, by shorting it, individual investors can only receive the premium equivalent to the bid price, which stands reasonably between the upper and lower bounds. Similar arguments can also pertain to the case when ask goes above lower bound, and bid sits beneath the lower bound.

To avoid any vagueness, this paper claims an option to be reasonably priced when both its bid and ask prices stand within the bounds. The duration of mispricings, as a result, is simply the span between the time when an option becomes mispriced for the first time, and the time when it subsequently turns back to be reasonably priced.

\subsection{Data}

The dataset in this study comes from Interactive Broker trading platform, which contains high frequency option bid-ask prices in a realistic trading environment from January 2011 to June 2012. Interactive Broker is one of the largest internet-based discount trading brokers in the world. With a real-time electronic trading platform, it provides live bid/ask quotes synchronized with AMEX, CBOE and other large exchanges. The dataset in this research comes from the historical snapshots of the quotes every fifteen minutes (See Figure 1 for an example). Due to limitation of the database, the sample period is restricted to half a year. However, the total observations amount to over 40,000 quotes.

This study choose S \& P 500 index ETF (SPY: NYSE) as the underlying security, as they have high liquidity and small bid-ask spread. The sample includes

\begin{tabular}{|c|c|c|c|c|c|}
\hline \multicolumn{5}{|c|}{ Call } & \multirow{2}{*}{ Description } \\
\hline Last & Change & Bid & Ask & Implied Vol. & \\
\hline C12.99 & & - 13.58 & 13.76 & N/A & 119 SMART \\
\hline C11.99 & & - 12.58 & 12.76 & $\mathrm{~N} / \mathrm{A}$ & 120 SMART \\
\hline C11.00 & & - 11.57 & 11.76 & $\mathrm{~N} / \mathrm{A}$ & 121 SMART \\
\hline C10.01 & & - 10.56 & 10.77 • & $\mathrm{N} / \mathrm{A}$ & 122 SMART \\
\hline 9.64 & +0.61 & - 9.64 & $9.74 \circ$ & $\mathrm{N} / \mathrm{A}$ & 123 SMART \\
\hline 8.71 & +0.64 & - 8.61 & $8.76 \circ$ & $43.182 \%$ & 124 SMART \\
\hline - 7.45 & +0.33 & - 7.57 & $7.74^{\circ}$ & N/A & 125 SMART \\
\hline - 6.70 & +0.49 & - 6.66 & $6.73 \bullet$ & $32.647 \%$ & 126 SMART \\
\hline - 5.63 & +0.40 & - 5.68 & $5.75 \bullet$ & $23.407 \%$ & 127 SMART \\
\hline - 4.65 & +0.38 & - 4.71 & $4.76 \bullet$ & $25.566 \%$ & 128 SMART \\
\hline - 3.78 & +0.42 & - 3.75 & $3.80 \circ$ & $26.198 \%$ & 129 SMART \\
\hline - 2.88 & +0.38 & - 2.83 & $2.89 \bullet$ & $25.282 \%$ & 130 SMART \\
\hline - 2.01 & +0.28 & 2.01 & $2.04^{\circ}$ & $23.330 \%$ & 131 SMART \\
\hline - 1.32 & +0.23 & - 1.29 & $1.31 \bullet$ & $22.501 \%$ & 132 SMART \\
\hline 0.74 & +0.14 & 0.72 & 0.73 。 & $21.087 \%$ & 133 SMART \\
\hline$\cdot 0.35$ & +006 & - 0.33 & 0.34 • & $20.096 \%$ & 134 SMART \\
\hline 0.12 & $434 \%-10: 03: 58$ & 0.12 & 0.13 & $19.593 \%$ & 135 SMART \\
\hline$\cdot 0.05$ & 0.00 & - 0.04 & 0.05 . & $19.889 \%$ & 136 SMART \\
\hline 0.02 & 0.00 & \multirow[t]{2}{*}{0.01} & $0.02 \circ$ & $20.819 \%$ & 137 SMART \\
\hline$\cdot 0.01$ & -0.01 & & 0.01 . & $22.768 \%$ & 138 SMART \\
\hline 0.01 & 0.00 & 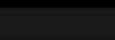 & 0.01 & $26.144 \%$ & 139 SMART \\
\hline \multirow[t]{2}{*}{0.01} & & & 0.01 & $29.494 \%$ & 140 SMART \\
\hline & & & 0.01 & $30.699 \%$ & 141 SMART \\
\hline
\end{tabular}

Figure 1. An snapshot example of live trading quotes. Source from Interactive Broker; 2012-06-27 at 10:02 am. 
options with various maturities, spanning from three months to three days. This research then applies option pricing bounds for each option at each time, in order to see the time series properties of mispricings.

The following filters apply to the dataset. Firstly, remove all quotes smaller than $\$ 0.05$ to reduce the tick size effects. Secondly, eliminate the options spanning over an ex-dividend day to address the differences between American and European calls. Lastly, check for potential entry errors such as nonmonotonic option premium as strike increases.

\subsection{Caveats}

Obviously, there are many other possible ways of estimating the statistical distribution of the S \& P 500 index returns other than these three models listed here. One caveat of the empirical results of "mispricings", as a result, is simply that the options market is priced with a different probability distribution than any of the three estimated probability distributions. Nevertheless, this may not be a major concern. I argue that if "mispricings" would result from inappropriate estimated return distribution, the identified "mispricings" should be quite frequent and persistent. Yet, the final results indicate the opposite. The last section will conduct further robustness checks on the issue.

Another noticeable concern is that the option pricing bounds stated in section 2 were derived specifically to price European options. Yet, the options on Index ETFs are American style. Although our option pricing bounds would underestimate the true price, several empirical designs may ease such concern. Note that an American call is identical to a European one if there is no dividend. Fortunately, the S \& P 500 index ETF has a schedule of dividend payment, i.e. approximately every three months. In order to eliminate the effect of dividend, the sample used in this study remove all options covering an ex-dividend date. Consequently, the resulted sample contains options with a maximum maturity of three months.

\section{Empirical Results}

This section describes the pattern of observed violations for the pricing bounds. The first part of the exercise identifies that more than one third of the options are mispriced, especially for out-of-money options. The next exercise find that the average duration of mispricing lasts around 5.5 trading hours, and the prices move in and out of the bounds frequently as maturity approaches.

\subsection{The Frequency of Violations}

Table 2 lists the pricing bounds along with the bid/ask prices for an option with a 12 days maturity on 5th July, 2012 at 10:19AM. For each different strike prices, the last column checks for the mispricing according to pricing bounds shown in Equation (1) and (2).

Table 3 summarizes the pattern of violations of the option pricing bounds 
Table 2. An example of the live quotes and pricing bounds.

\begin{tabular}{|c|c|c|c|c|c|c|}
\hline Strike & Upper bound & Lower bound & Bid & Ask & Over price & Under price \\
\hline 125 & 11.52557 & 11.43186 & 11.37 & 11.59 & FALSE & FALSE \\
\hline 126 & 10.5198 & 10.43053 & 10.37 & 10.58 & FALSE & FALSE \\
\hline 127 & 9.512736 & 9.438168 & 9.37 & 9.57 & FALSE & FALSE \\
\hline 128 & 8.507658 & 8.431843 & 8.36 & 8.56 & FALSE & FALSE \\
\hline 129 & 7.501162 & 7.435464 & 7.36 & 7.54 & FALSE & FALSE \\
\hline 130 & 6.494515 & 6.440102 & 6.36 & 6.51 & FALSE & FALSE \\
\hline 131 & 5.48881 & 5.438257 & 5.36 & 5.52 & FALSE & FALSE \\
\hline 132 & 4.489149 & 4.401126 & 4.45 & 4.51 & FALSE & FALSE \\
\hline 133 & 3.496384 & 3.444487 & 3.49 & 3.54 & FALSE & FALSE \\
\hline 134 & 2.538595 & 2.451742 & 2.56 & 2.59 & TRUE & FALSE \\
\hline 135 & 1.691972 & 1.513852 & 1.68 & 1.7 & FALSE & FALSE \\
\hline 136 & 0.992089 & 0.737409 & 0.94 & 0.95 & FALSE & FALSE \\
\hline 137 & 0.501882 & 0.254373 & 0.4 & 0.42 & FALSE & FALSE \\
\hline 138 & 0.212261 & 0.050712 & 0.12 & 0.13 & FALSE & FALSE \\
\hline 139 & 0.074505 & 0 & 0.03 & 0.04 & FALSE & FALSE \\
\hline 140 & 0.021266 & 0 & 0.01 & 0.02 & FALSE & FALSE \\
\hline 141 & 0.00486 & 0 & N/A & 0.01 & FALSE & FALSE \\
\hline 142 & 0.009698 & 0 & N/A & 0.01 & FALSE & FALSE \\
\hline 143 & 0.001851 & 0 & N/A & 0.01 & FALSE & FALSE \\
\hline 144 & 0.005843 & 0 & N/A & 0.01 & FALSE & FALSE \\
\hline 145 & 0.004847 & 0 & N/A & 0.01 & FALSE & FALSE \\
\hline 146 & 0.001962 & 0 & N/A & 0.01 & FALSE & FALSE \\
\hline 147 & 0.012707 & 0 & N/A & 0 & FALSE & FALSE \\
\hline 148 & 0.001173 & 0 & N/A & 0 & FALSE & FALSE \\
\hline 149 & 0.006967 & 0 & N/A & 0 & FALSE & FALSE \\
\hline 150 & 0.002497 & 0 & N/A & 0 & FALSE & FALSE \\
\hline
\end{tabular}

*Source: Data retrieved from Interactive Broker on 5th July, 2012 at 10:19AM. The underlying (SPY) prices were 136.46 then. The option will expire on 20th July, 2012. The upper and lower bounds are calculated according to the $\operatorname{ARMA}(1,1)-\mathrm{GARCH}(1,1)$ approach.

from July 2011 to July 2012. The violations are displayed as the percentage of the total number of quotes in each moneyness range. While a majority of the quotes are reasonably priced, on average, $19.53 \%$ of the quotes are overpriced and $6.33 \%$ of the quotes are underpriced according to the bounds. The larger proportion of violating upper bounds suggests the options have a tendency to be costly. Similar to Czerwonko, Jackwerth, and Perrakis [5], the results show that a majority of the identified overpricing are OTM options, regardless of the methods of estimating the bounds. This implies that any risk-averse trader can 
Table 3. Percentage of violations of the pricing bounds out of the total number of Quotes: SPY.

\begin{tabular}{|c|c|c|c|c|c|}
\hline \multirow[t]{2}{*}{ Model } & \multicolumn{4}{|c|}{ Moneyness $(K / P)$} & \multirow[t]{2}{*}{ Total (\%) } \\
\hline & $0.96-0.99$ & $0.99-1.01$ & $1.01-1.03$ & $1.03-1.05$ & \\
\hline \multicolumn{6}{|l|}{ Bootstrapping } \\
\hline Overpricing & 0.80 & 3.12 & 14.19 & 5.29 & 23.40 \\
\hline Underpricing & 1.81 & 3.84 & 0.13 & 0.05 & 5.83 \\
\hline \multicolumn{6}{|l|}{$A R M A-G A R C H$} \\
\hline Overpricing & 0.19 & 1.82 & 9.20 & 4.37 & 15.58 \\
\hline Underpricing & 1.79 & 3.96 & 0.36 & 0.30 & 6.41 \\
\hline \multicolumn{6}{|l|}{ Adjusted VIX } \\
\hline Overpricing & 0.93 & 2.85 & 11.28 & 4.58 & 19.65 \\
\hline Underpricing & 1.92 & 3.63 & 0.04 & 0.10 & 5.70 \\
\hline \multicolumn{6}{|l|}{ Average } \\
\hline Overpricing & 0.64 & 2.60 & 11.55 & 4.74 & 19.53 \\
\hline Underpricing & 1.84 & 3.81 & 0.53 & 0.15 & 6.33 \\
\hline
\end{tabular}

*Source: Author's computation. The table displays the percentages of bid/ask quotes violating the pricing bounds out of the total number of observed quotes under different estimation methods. The under pricing is defined as ask price < lower bound, and overpricing is identified if bid price $>$ upper bound. Numbers are in percentage.

improve expected utility by writing those "mispriced" call options net of transaction costs and bid-ask spread.

An experiment not shown in Table 3 indicates that the typical violation size of the bounds is between $\$ 0.01$ to $\$ 0.05$ for $65 \%$ of the total violations. The violations are widespread, with a proportion of approximately $30 \%$, when maturity approaches (less than one week).

\subsection{The Duration of Violations}

This section further investigates the time series properties of violations of pricing bounds. Specifically, Table 4 illustrates the average duration of the violations under different estimation approaches for options with different time to maturity $(T-t)$.

On average, the duration of a mispricing persists less than two trading days. This implies that the majority of the mispricings disappear in a short period of time, which refutes the prediction of a persistent model misspecification. The bootstrapping method identifies a longer duration than others as it usually produces wider bounds.

Strikingly, although the violations of the lower bounds are only occasional, they are likely to be more persistent than overpricing. This suggests a possible misspecification of the lower bounds. Moreover, as the maturity date comes closer, the interval of the mispricings period diminishes considerably, from around 13 trading hours to 4 hours, regardless of the methodology to estimate 
Table 4. Average duration of violating the pricing bounds (in Trading Hours).

\begin{tabular}{ccccc}
\hline Model & \multicolumn{4}{c}{ Time to Maturity $(T-t)$} \\
\hline Bootstrapping & $>1$ Month & 2 to 3 Weeks & 1 to 2 Weeks & $<1$ Week \\
Overpricing & 16.51 & 15.90 & 8.67 & 5.30 \\
Underpricing & 17.13 & 15.44 & 9.33 & 7.79 \\
ARMA-GARCH & & & & \\
Overpricing & 12.63 & 11.40 & 6.24 & 3.77 \\
Underpricing & 13.32 & 11.25 & 6.75 & 5.74 \\
Adjusted VIX & & & & \\
Overpricing & 10.83 & 9.19 & 6.73 & 2.79 \\
Underpricing & 11.17 & 9.25 & 6.64 & 4.54 \\
\hline
\end{tabular}

*Source: Author's computation. This table illustrates the average duration of the violations under different estimation approaches for options with different time to maturity $(T-t)$. The duration of a mispricing is defined as the span between the time when an option becomes mispriced for the first time, and the time when it subsequently turns back to be reasonably priced. The claim of a reasonably priced option represents the case when both its bid and ask prices stand within the bounds.

the bounds. These agree with the arguments pinpointing the irrationality of investors shortly before maturity [10].

As argued before, the "mispricings" may be either the results of model misspecifications or temporary irrationality of investors. Our primary results indicate that violations of the pricing bounds are typically short-lived. These results favor the later hypothesis, supporting the option pricing bounds derived in Constantinides and Perrakis [7].

\subsection{Discussion}

This section conducts several robustness checks which may undermine the results.

In the first place, the short-lived violations may purely results from overestimation of upper bounds or underestimate the lower bounds. For example, the upper bounds could be so high that only sporadic extreme market fluctuations are documented. To ease this concern, this paper manually adjusts the pricing bounds downwards. The results show that only when decreasing the bounds by as much as $\$ 0.15$ could we observe an average mispricing duration of five trading days.

In addition, the Monte Carlo simulation to calculate the pricing bounds may also lead to frequent short term violations of the bounds, as the bounds fluctuate across time simply because of the sampling errors. To address this issue, the simulation in this paper employs a fixed seed in the random number generator. Thus, this procedure could only bias the duration of the violations upward, as the sampling errors persist through time. 


\section{Conclusions}

A number of literatures have documented evidences suggesting the mispricing of options. Since it is hard to believe the markets are inefficient for a long term, the observed "mispricing" might either result from transitory market inefficiency or from model misspecifications.

After constructing option pricing bounds based on stochastic dominance, this paper examines the time series properties of option mispricings using high frequency bid-ask quotes. This study contributes to the literature by showing that most violations of the stochastic dominance upper bounds of Constant inides and Perrakis [7] last no more than 10 trading hours. The results imply that the observed widespread mispricing in options might be the result of temporary inefficiency (e.g. transaction costs, overreaction, liquidity etc.) rather than a model misspecification, such as estimation biases of the parameters, or an overlooked persistent risk factor.

Possible extension that could substantiate the results obtained in this paper could be establishing a high-frequency trading rule and testing its profitability. As the results of this paper suggest that the mispricings are mostly short-lived, traders could profit from fast convergence of mispriced options. Another possible future improvement could be including high-frequency quotes for a longer sample period. I leave these extensions to future endeavors.

\section{References}

[1] Rubinstein, M. (1994) Implied Binomial Trees. Journal of Finance, 49, 771-818. https://doi.org/10.1111/j.1540-6261.1994.tb00079.x

[2] Jackwerth, J. and Rubinstein, M. (1996) Recovering Probability Distributions from Option Prices. Journal of Finance, 51, 1611-1632. https://doi.org/10.1111/j.1540-6261.1996.tb05219.x

[3] Coval, J. and Shumway, T. (2001) Expected Option Returns. Journal of Finance, 59, 983-1009. https://doi.org/10.1111/0022-1082.00352

[4] Bakshi, G. and Kapadia, N. (2003) Delta-Hedged Gains and the Negative Market Volatility Risk Premium. Review of Financial Studies, 16, 527-566. https://doi.org/10.1093/rfs/hhg002

[5] Constantinides, G., Czerwonko, M., Jackwerth, J. and Perrakis, S. (2011) Are Options on Index Futures Profitable for Risk-Averse Investors? Empirical Evidence. Journal of Finance, 66, 1407-1437. https://doi.org/10.1111/j.1540-6261.2011.01665.x

[6] Constantinides, G., Jackwerth, J. and Perrakis, S. (2009) Mispricing of S \& P 500 Index Options. Review of Financial Studies, 22, 1247-1277. https://doi.org/10.1093/rfs/hhn009

[7] Constantinides, G. and Perrakis, S. (2007) Stochastic Dominance Bounds on American Option Prices in Markets with Frictions. Review of Finance, 11, 71-115. https://doi.org/10.1093/rof/rfl001

[8] Santa-Clara, P. and Saretto, A. (2009) Option Strategies: Good Deals and Margin Calls. Journal of Financial Markets, 12, 391-417. https://doi.org/10.1016/j.finmar.2009.01.002

[9] Constantinides, G. and Perrakis, S. (2002) Stochastic Dominance Bounds on Deriv- 
atives Prices in a Multiperiod Economy with Proportional Transaction Costs. Journal of Economic Dynamics and Control, 26, 1323-1352.

https://doi.org/10.1016/S0165-1889(01)00047-1

[10] Poteshman, A. and Serbin, V. (2003) Clearly Irrational Financial Market Behavior: Evidence from the Early Exercise of Exchange Traded Stock Options. Journal of Finance, 58, 37-70. https://doi.org/10.1111/1540-6261.00518 